\title{
PENCILS OF EULER TRIPLES, II
}

\author{
ZVONKO ČERIN
}

\begin{abstract}
In this second part of the paper [7] we will continue to study the families of triples $(u, v, w)$ of elements in a commutative ring $R$ with the property that $v w+n=\widetilde{u}^{2}, w u+n=\widetilde{v}^{2}$ and $u v+n=\widetilde{w}^{2}$ for some $n, \widetilde{u}, \widetilde{v}, \widetilde{w} \in R$. They are built from the Euler triples $(a, b, a+b \pm 2 t)$, where $n=t^{2}-a b$. The idea is to use an extra element $m \in R$ and take either

$$
A=\left(a, a m^{2}+2 t m+b, a m^{2}+2(t+a) m+a+b+2 t\right)
$$

or

$$
B=\left(a, a m^{2}+2 t m+b, a m^{2}+2(t-a) m+a+b-2 t\right) .
$$

In this way we get the so called pencils that are here further explored. Many properties and identities for their components and various sums and products are established. The associated pencils $\widetilde{A}$ and $\widetilde{B}$ build from the triples $(\widetilde{u}, \widetilde{v}, \widetilde{w})$ are also studied. We present various methods how one can get squares and cubes from the four pencils, describe values of determinants and generalized determinants of matrices with rows from the pencils and give factorizations of some expressions from members of the pencils $A, B, \widetilde{A}$ and $\widetilde{B}$ and examine the role of the parameter $m$.
\end{abstract}

\section{INTRODUCTION}

In this introduction we recall some highlights from the introduction of the first part [7] in order to make connection easier.

The subject matter of both papers are $S(n)$-m-tuples in a commutative ring $R[9]$. The products of pairs of its different components increased by an $n \in R$ is always a square. They appeared already in the works of the Greek mathematician Diophantus of Alexandria who found that $\mathfrak{d}=\left(\frac{1}{16}, \frac{33}{16}, \frac{17}{4}, \frac{105}{16}\right)$ is the $S(1)$-quadruple of rationals (see [3] and [1]).

Starting from the Euler $S(n)$-triples $(a, b, a+b \pm 2 t)$, for $a, b, t \in R$ and $n=t^{2}-a b$, we consider the functions $\alpha, \beta: R^{4} \rightarrow R^{3}$ called the pencils of

2000 Mathematics Subject Classification. Primary 11B37, 11B39, 11 D09.

Key words and phrases. Ring, squares, $S(n)$-triple, Euler triple, pencil of Euler triples, basic symmetric functions, determinants, generalized determinants, vector cross-product. 
Euler $S(n)$-triples and defined as follows. For $a, b, t, m \in R$, let $\alpha(a, b, t, m)$ and $\beta(a, b, t, m)$ be the triples $A$ and $B$ with components $A_{1}=B_{1}=a$, $A_{2}=B_{2}=a m^{2}+2 t m+b, A_{3}=a m^{2}+2(t+a) m+a+b+2 t$ and $B_{3}=$ $a m^{2}+2(t-a) m+a+b-2 t$.

The extend of values of $\alpha$ and $\beta$ is illustrated by the $S(1)$-triples $\left(F_{2 n}\right.$, $\left.F_{2 n+2}, F_{2 n+4}\right)$ of three consecutive Fibonacci numbers with even indices. When $n=1$, we have the triple $\mathfrak{r}=(1,3,8)$ (that can be enlarged with the number 120 to the famous Fermat $S(1)$-quadruple $\mathfrak{F}=(1,3,8,120)$ [2]). Using the above blowing up method, from the triple $\mathfrak{r}$, we get the pencils $\mathfrak{a}=\mathfrak{a}(m)=\left(1, m^{2}+4 m+3, m^{2}+6 m+8\right)$ and $\mathfrak{b}=\mathfrak{b}(m)=\left(1, m^{2}+4 m+\right.$ $\left.3, m^{2}+2 m\right)$ of infinitely many $S(1)$-triples like $\mathfrak{a}(2)=(1,15,24), \mathfrak{a}\left(\frac{1}{2}\right)=(1$, $\left.\frac{21}{4}, \frac{45}{4}\right)$ and $\mathfrak{b}(-10)=(1,63,80)$. Also, $\mathfrak{b}(3+2 i)=(1,20+20 i, 11+16 i)$ (since the parameter $m$ can even be a complex number; $i=\sqrt{-1}$ ).

The Euler $S(n)$-triples are quite common among the $S(n)$-triples. They are usually the first three terms of an $S(n)$-quadruple whose fourth component can be figured out from the initial Euler triple. For example, the following could be located in the reference [10, Theorems 3-7, Example 1, Corollaries 2 and 3] and the Jones polynomial Euler $S(1)$-triple $(n, n+2,4(n+1))$ that includes for $n=\frac{1}{16}$ the first three terms of $\mathfrak{d}$ (see [12] and [13]).

Some of our results have been discovered earlier for the Euler $S(-4)$-triples and the $S(8)$-triples from the Pell and the Pell-Lucas numbers in [4] and for the pencils on these triples in [5] and for the pencils built on the $S(-1)$-triples and the $S(5)$-triples from the Fibonacci and the Lucas numbers in [6].

The present paper further investigates the common properties of all pencils of Euler $S(n)$-triples (like $\mathfrak{a}$ and $\mathfrak{b}$ and all others listed above). In another paper [8], the author will consider similar blowing up procedure for the Euler $S(n)$-quadruples.

The present paper, like its first part [7], searches for the additional (intrinsic) properties that a given pencil of Euler $S(n)$-triples might have besides its existence and the defining property. The goal is to explore the properties of the pencils $\alpha$ and $\beta$ and to understand better their structure.

In order to help the reader follow our notation and counter the dry abstract ring setting, we give many concrete examples for the favorite triple $\mathfrak{r}=(1,3,8)$ and the associated pencils $\mathfrak{a}$ and $\mathfrak{b}$ of $S(1)$-triples. The notation and the numeration of the properties continues from the first part [7].

\section{The Linear SUMS OF COMPONENTS}

It follows easily from the Properties 4 and 5 that $E_{\sigma_{1}}=2\left(\widetilde{E}_{1}+a\right)$, $\widetilde{A}_{\sigma_{1}}=A_{3}+\widetilde{E}_{3}, \widetilde{B}_{\sigma_{1}}=E_{2}+\widetilde{B}_{2}, A_{\sigma_{1}^{*}}=2 \widetilde{A}_{2}, B_{\sigma_{1}^{*}}=-2 \widetilde{B}_{2}, \widetilde{A}_{\sigma_{1}^{*}}=\widetilde{A}_{1}-a$ and $\widetilde{B}_{\sigma_{1}^{*}}=\widetilde{B}_{1}+a=E_{2}-\widetilde{B}_{2}=B_{3}+\widetilde{E}_{3}$. Somewhat less obvious representations for these sums are the following. 
Property 29. For $a, b, t, m, u \in R$, if $a u=2 \widetilde{E}_{3}$, then the sum $\widetilde{A}_{\sigma_{1}}$ is equal $E_{2}+(m+u)_{2}^{\widetilde{A}}$. If $R$ has the multiplicative identity e, then $\widetilde{E}_{\sigma_{1}}=(m+e)_{1}^{\widetilde{E}}$ $-a$.

Proof. Let $a u=2 \widetilde{E}_{3}=2(a m+t)$. The sum $\widetilde{A}_{\sigma_{1}}-E_{2}-(m+u)_{2}^{\widetilde{A}}$ is $2(a m$ $+t)-a u=0$. Hence, $\widetilde{A}_{\sigma_{1}}=E_{2}+(m+u)_{2}^{\widetilde{A}}$.

Let $E=A$. Since we have $\widetilde{A}_{\sigma_{1}}=a m^{2}+(3 a+2 t) m+a+b+3 t$ and $(m+e)_{1}^{\widetilde{A}}=a m^{2}+(3 a+2 t) m+2 a+b+3 t$, it follows that $\widetilde{A}_{\sigma_{1}}$ is the difference $(m+e)_{1}^{\widetilde{A}}-a$.

For the triple $\mathfrak{a}$, the first identity in Property 30 is (with $u=2 m+4$ )

$$
\widetilde{\mathfrak{a}}_{\sigma_{1}}=m^{2}+7 m+10=\left(m^{2}+4 m+3\right)+(3 m+7)=\mathfrak{a}_{2}+(m+u)_{2}^{\tilde{\mathfrak{a}}} .
$$

Property 30. If $R$ is a field, then for $a, b, t, k, m \in R$, the sum $\widetilde{A}_{\sigma_{1}}$ is either $E_{2}+3\left(m-\frac{2}{3}\right)_{2}^{\widetilde{A}},\left(m+\frac{3}{2}\right)_{2}^{E}-\frac{5}{4} a$ or $\widetilde{A}_{1}+2\left(m-\frac{1}{2}\right)_{2}^{\widetilde{E}}$ and $\widetilde{B}_{\sigma_{1}}$ is either $B_{3}+3\left(m-\frac{2}{3}\right) \frac{\widetilde{E}}{3},\left(m+\frac{3}{2}\right)_{3}^{B}-\frac{5}{4} a$ or $\widetilde{B}_{1}+2\left(m+\frac{1}{2}\right)_{2}^{\widetilde{B}}$.

Proof. Since the product $3\left(m-\frac{2}{3}\right)_{3}^{\widetilde{E}}$ is $3 a m-2 a+t$ and $B_{3}$ is the sum $a m^{2}-2(a-t) m+a+b$, their sum is $a m^{2}+(a+2 t) m-a+t+b$ that we recognize as $\widetilde{B}_{\sigma_{1}}$.

Property 31. If the ring $R$ has the multiplicative identity $e$, then for $a, b, t$, $m \in R$, it holds $\widetilde{A}_{\sigma_{1}^{*}}=E_{2}+(m-2 e)_{2}^{\widetilde{A}}=A_{3}-(m+2 e)_{3}^{\widetilde{E}}$.

Proof. Since $E_{2}$ is $a m^{2}+2 t m+b$ and $(m-2 e)_{2}^{\widetilde{A}}$ is $a m-a+t$ their sum is $a m^{2}+(a+2 t) m-a+b+t$ that we recognize as $\widetilde{A}_{\sigma_{1}^{*}}$.

The next item considers sums and differences of various sums of $A$ and $B$ and $\widetilde{A}$ and $\widetilde{B}$. These identities are straightforward consequences of the previous properties.

Property 32. For $a, b, t, m \in R$,

$$
\begin{gathered}
A_{\sigma_{1}}-B_{\sigma_{1}}=A_{\sigma_{1}^{*}}-B_{\sigma_{1}^{*}}=4 \widetilde{E}_{3}, \quad \widetilde{A}_{\sigma_{1}}-\widetilde{B}_{\sigma_{1}}=2 \widetilde{A}_{2}, \\
A_{\sigma_{1}}+B_{\sigma_{1}}=4\left(a+E_{2}\right), \quad \widetilde{A}_{\sigma_{1}}+\widetilde{B}_{\sigma_{1}}=2\left(E_{2}+2 \widetilde{E}_{3}\right)=2\left(A_{3}-a\right), \\
A_{\sigma_{1}^{*}}+B_{\sigma_{1}^{*}}=4 a, \quad \widetilde{A}_{\sigma_{1}^{*}}+\widetilde{B}_{\sigma_{1}^{*}}=2 E_{2}, \quad \widetilde{A}_{\sigma_{1}^{*}}-\widetilde{B}_{\sigma_{1}^{*}}=2 \widetilde{B}_{2} .
\end{gathered}
$$

3. The quadratic sums of COMPOnents

In the Property 9 we got formulae for $A_{\sigma_{2}}$ and $B_{\sigma_{2}}$. We want now analogous formulae for $\widetilde{A}_{\sigma_{2}}$ and $\widetilde{B}_{\sigma_{2}}$. In the next property, adding and subtracting $n$ from $\widetilde{E}_{\sigma_{2}}$ leads to factorizations with 2 as a factor. For the triple $\mathfrak{r}$, we have $\widetilde{\mathfrak{a}}_{\sigma_{2}}-1=(3 \cdot 2+2 \cdot 5+5 \cdot 3)-1=30=2 \cdot 5 \cdot 3=2 \widetilde{\mathfrak{a}}_{1} \widetilde{\mathfrak{a}}_{2}$. 
Property 33. For $a, b, t, m \in R$, the following is true:

$$
\widetilde{A}_{\sigma_{2}}=2 \widetilde{A}_{1} \widetilde{A}_{2}+n=2 A_{3} \widetilde{E}_{3}-n, \quad \widetilde{B}_{\sigma_{2}}=2 \widetilde{B}_{1} \widetilde{E}_{3}+n=2 E_{2} \widetilde{B}_{2}-n .
$$

Proof. The difference $\widetilde{A}_{\sigma_{2}}-2 \widetilde{A}_{1} \widetilde{A}_{2}$ is $t^{2}-a b$. Since $t^{2}-a b=n$, the first formula holds.

Similarly, the difference $\widetilde{B}_{\sigma_{2}}-2 E_{2} \widetilde{B}_{2}$ is $a b-t^{2}$. Since $a b-t^{2}=-n$, the last formula also holds.

The factorization occurs also when $a^{2}$ is subtracted or when $5 a^{2}-3 n$ is added to the sum $E_{\sigma_{2}}+\widetilde{E}_{\sigma_{2}}$. For the triple $\mathfrak{r}$, the third identity is $\mathfrak{r}_{\sigma_{2}}+\widetilde{\mathfrak{r}}_{\sigma_{2}}+2=35+31+2=68=4 \cdot 17=\left(\mathfrak{r}_{2}+1\right)\left(\mathfrak{r}_{2}+4 \cdot \widetilde{\mathfrak{r}}_{2}+2\right)$.

Property 34. For $a, b, t, m \in R$, the following is true:

$$
\begin{gathered}
A_{\sigma_{2}}+\widetilde{A}_{\sigma_{2}}-a^{2}=\left(E_{2}+\widetilde{E}_{3}\right)\left(A_{3}+\widetilde{A}_{2}+2 a\right), \\
B_{\sigma_{2}}+\widetilde{B}_{\sigma_{2}}-a^{2}=\left(E_{2}-\widetilde{E}_{3}\right)\left(B_{3}+3 \widetilde{E}_{3}+a\right), \\
A_{\sigma_{2}}+\widetilde{A}_{\sigma_{2}}+5 a^{2}-3 n=\left(E_{2}+a\right)\left(E_{2}+4 \widetilde{A}_{2}+2 a\right), \\
B_{\sigma_{2}}+\widetilde{B}_{\sigma_{2}}+5 a^{2}-3 n=\left(B_{3}+a\right)\left(B_{3}+4 \widetilde{E}_{3}+2 a\right) .
\end{gathered}
$$

Proof. The left hand side $A_{\sigma_{2}}+\widetilde{A}_{\sigma_{2}}-a^{2}$ of the first relation factors into the product of $f=a m^{2}+(a+2 t) m+b+t$ and $g=a m^{2}+(3 a+2 t) m+4 a+$ $3 t+b$. But, $f$ is $E_{2}+\widetilde{E}_{3}$ and $g$ is $A_{3}+\widetilde{A}_{2}+2 a$.

In the next item we take sums that use all four sums $E_{\sigma_{2}}$ and $\widetilde{E}_{\sigma_{2}}$. Their representations are similar and the factor 2 appears in both sums.

Property 35. For $a, b, t, m \in R$,

$$
\begin{gathered}
A_{\sigma_{2}}+\widetilde{A}_{\sigma_{2}}+B_{\sigma_{2}}+\widetilde{B}_{\sigma_{2}}=2\left[\left(E_{2}+\widetilde{A}_{2}\right)^{2}+a\left(E_{2}-\widetilde{E}_{3}\right)\right], \\
A_{\sigma_{2}}-\widetilde{A}_{\sigma_{2}}+B_{\sigma_{2}}-\widetilde{B}_{\sigma_{2}}=2\left[\left(E_{2}-\widetilde{A}_{2}\right)^{2}+3 a\left(E_{2}-\widetilde{E}_{3}\right)-2 n\right] .
\end{gathered}
$$

Proof. Since $A_{\sigma_{2}}+\widetilde{A}_{\sigma_{2}}+B_{\sigma_{2}}+\widetilde{B}_{\sigma_{2}}-2\left(E_{2}+\widetilde{A}_{2}\right)^{2}$ is the product of $2 a$ and $a m^{2}+2 t m+b-a m-t=E_{2}-\widetilde{E}_{3}$, the first identity follows.

Now we move to the second alternating symmetric sum $\sigma_{2}^{*}$. The next are the formulas for $E_{\sigma_{2}^{*}}$ and $\widetilde{E}_{\sigma_{2}^{*}}$. Subtracting $a^{2}$ from $E_{\sigma_{2}^{*}}$ leads to the factorization while the $n=t^{2}-a b$ plays important role for $\widetilde{E}_{\sigma_{2}^{*}}$.

Property 36. For $a, b, t, m \in R$,

$$
\begin{gathered}
A_{\sigma_{2}^{*}}-a^{2}=\left(E_{2}-a\right)\left(\widetilde{A}_{1}+\widetilde{A}_{2}+a\right), \quad \widetilde{A}_{\sigma_{2}^{*}}=2 a \widetilde{A}_{1}+n, \\
B_{\sigma_{2}^{*}}-a^{2}=\left(E_{2}-a\right)\left(\widetilde{B}_{1}-\widetilde{B}_{2}+a\right), \quad \widetilde{B}_{\sigma_{2}^{*}}=n .
\end{gathered}
$$


Proof. The difference $\widetilde{A}_{\sigma_{2}^{*}}-a^{2}$ factors as the product $f g$ of $f=\left(a m^{2}+\right.$ $2 t m+b)-a=E_{2}-a$ and $g=\left(a m^{2}+(a+2 t) m+b+t\right)+(a m+a+t)$ $+a=\widetilde{A}_{1}+\widetilde{A}_{2}+a$. Hence, the first identity holds.

For the second identity, notice that the difference $\widetilde{A}_{\sigma_{2}^{*}}-2 \widetilde{A}_{1}$ is the familiar $t^{2}-a b=n$.

Notice that the last equality in the above property was already noted in the Property 23 in different context.

We continue by showing that the sums $E_{\sigma_{2}^{*}}+\widetilde{E}_{\sigma_{2}^{*}}$ have quite similar representations.

Property 37. For all $a, b, t, m \in R$,

$$
A_{\sigma_{2}^{*}}+\widetilde{A}_{\sigma_{2}^{*}}=\left(\widetilde{A}_{1}+a\right)^{2}-2 a \widetilde{A}_{2}, \quad B_{\sigma_{2}^{*}}+\widetilde{B}_{\sigma_{2}^{*}}=\left(\widetilde{B}_{1}+a\right)^{2}-2 a B_{3} .
$$

Proof. Since $A_{\sigma_{2}^{*}}+\widetilde{A}_{\sigma_{2}^{*}}+2 a \widetilde{A}_{2}$ is the square of $a m^{2}+(a+2 t) m+b+t+$ $a=\widetilde{A}_{1}+a$, the first identity holds.

We proceed with the version of the Property 34 for the sum $\sigma_{2}^{*}$. For the triple $\mathfrak{r}$, the first identity is $\mathfrak{r}_{\sigma_{2}^{*}}+\widetilde{\mathfrak{r}}_{\sigma_{2}^{*}}=19+11=30=3 \cdot(8+2 \cdot 1)$.

Property 38. For all $a, b, t, m \in R$,

$$
A_{\sigma_{2}^{*}}+\widetilde{A}_{\sigma_{2}^{*}}+a^{2}-n=A_{2}\left(A_{3}+2 a\right), \quad B_{\sigma_{2}^{*}}+\widetilde{B}_{\sigma_{2}^{*}}-a^{2}-n=\left(B_{2}-a\right)\left(B_{3}+a\right) .
$$

Proof. For the proof of the second identity, use the values for $B_{\sigma_{2}^{*}}$ and $\widetilde{B}_{\sigma_{2}^{*}}$ from the Property 36 and use $B_{3}=\widetilde{B}_{1}-\widetilde{B}_{2}$.

The sum $\sigma_{3}$ does not give any interesting properties except that the identities $\widetilde{A}_{\sigma_{3}}-A_{\sigma_{3}}=n\left(A_{2}+\widetilde{A}_{2}\right)$ and $\widetilde{B}_{\sigma_{3}}-B_{\sigma_{3}}=n\left(B_{3}+\widetilde{B}_{3}\right)$ show that $n$ is a factor of $\widetilde{E}_{\sigma_{3}}-E_{\sigma_{3}}$.

\section{The PRODUCTS OF SUMS}

The products of the linear sums of components also give interesting relationships and even complete squares.

Property 39. For $a, b, t, m \in R$,

$$
\begin{gathered}
A_{\sigma_{1}} A_{\sigma_{1}^{*}}-4 E_{2} \widetilde{A}_{2}=4 \widetilde{A}_{2}^{2}, \quad B_{\sigma_{1}} B_{\sigma_{1}^{*}}+4 E_{2} \widetilde{B}_{2}=4 \widetilde{B}_{2}^{2}, \\
A_{\sigma_{1}} B_{\sigma_{1}}-4 A_{3} B_{3}=12 \widetilde{E}_{3}^{2}, \quad A_{\sigma_{1}^{*}} B_{\sigma_{1}^{*}}+4 \widetilde{E}_{3}^{2}=4 a^{2}, \\
\widetilde{A}_{\sigma_{1}} \widetilde{A}_{\sigma_{1}^{*}}=A_{3}\left(A_{3}-3 a\right)+a^{2}-n, \quad \widetilde{B}_{\sigma_{1}} \widetilde{B}_{\sigma_{1}^{*}}=A_{3}\left(B_{3}+a\right)-3\left(a^{2}-n\right) .
\end{gathered}
$$

Proof. The difference $\widetilde{A}_{\sigma_{1}} \widetilde{A}_{\sigma_{1}^{*}}-A_{3}\left(A_{3}-3 a\right)$ simplifies to $a^{2}-n$ while the difference $\widetilde{B}_{\sigma_{1}} \widetilde{B}_{\sigma_{1}^{*}}-A_{3}\left(B_{3}+a\right)$ reduces to $-3\left(a^{2}-n\right)$. 
In the next two properties we show that certain sums of products of symmetric sums of the triples $E$ and $\widetilde{E}$ have interesting representations as products.

Property 40. For $a, b, t, m \in R$,

$$
A_{\sigma_{1}^{*}} B_{\sigma_{1}}+A_{\sigma_{1}} B_{\sigma_{1}^{*}}=8\left(a^{2}-n\right), \quad A_{\sigma_{1}^{*}} B_{\sigma_{1}}-A_{\sigma_{1}} B_{\sigma_{1}^{*}}=8 E_{2} \widetilde{E}_{3} .
$$

Proof. Since $A_{\sigma_{1}^{*}} B_{\sigma_{1}}=4 \widetilde{A}_{2}\left(\widetilde{B}_{1}+a\right)$ and $A_{\sigma_{1}} B_{\sigma_{1}^{*}}=-4 \widetilde{B}_{2}\left(\widetilde{A}_{1}+a\right)$, the sum $A_{\sigma_{1}^{*}} B_{\sigma_{1}}+A_{\sigma_{1}} B_{\sigma_{1}^{*}}$ is $8\left(a^{2}-n\right)$, while the difference simplifies to $8 E_{2} \widetilde{E}_{3}$.

Property 41. For $a, b, t, m \in R$, the following is true:

$$
\begin{gathered}
A_{\sigma_{3}} B_{\sigma_{1}}-B_{\sigma_{3}} A_{\sigma_{1}}=4 a E_{2} \widetilde{E}_{3}\left(E_{2}+a\right), \\
B_{\sigma_{3}} A_{\sigma_{1}^{*}}-A_{\sigma_{3}} B_{\sigma_{1}^{*}}=4 a E_{2} \widetilde{E}_{3}\left(E_{2}-a\right), \\
A_{\sigma_{3}} B_{\sigma_{2}}-B_{\sigma_{3}} A_{\sigma_{2}}=A_{\sigma_{3}} B_{\sigma_{2}^{*}}-B_{\sigma_{3}} A_{\sigma_{2}^{*}}=4 a^{2} E_{2}^{2} \widetilde{E}_{3} .
\end{gathered}
$$

Proof. In order to prove the first formula, note that $A_{1}=B_{1}=a$ and $A_{2}$ $=B_{2}=E_{2}$. The left hand side is thus the product $a E_{2}$ multiplied by $B_{3} A_{\sigma_{1}^{*}}-A_{3} B_{\sigma_{1}^{*}}=4 \widetilde{E}_{3}\left(E_{2}-a\right)$.

\section{THE POWERS OF THE SUMS}

In this section we give two sets of identities for some symmetric expressions that use the powers (second, third and fourth) of the sums $\sigma_{1}$ and $\sigma_{1}^{*}$ of the four pencils $\alpha, \beta, \widetilde{\alpha}$ and $\widetilde{\beta}$. We show that these expressions have long factorizations.

Property 42. For all $a, b, t, m \in R$,

$$
\begin{gathered}
A_{\sigma_{1}}^{2}+B_{\sigma_{1}}^{2}-A_{\sigma_{1}^{*}}^{2}-B_{\sigma_{1}^{*}}^{2}=8 E_{2}\left(E_{2}+2 a\right), \\
A_{\sigma_{1}}^{3}+B_{\sigma_{1}}^{3}-A_{\sigma_{1}^{*}}^{3}-B_{\sigma_{1}^{*}}^{3}=16 E_{2}\left[E_{2}^{2}+3 a\left(2 E_{2}+a\right)+3 n\right], \\
A_{\sigma_{1}}^{4}+B_{\sigma_{1}}^{4}-A_{\sigma_{1}^{*}}^{4}-B_{\sigma_{1}^{*}}^{4}=32 E_{2}\left(E_{2}+2 a\right)\left[E_{2}^{2}+2 a\left(4 E_{2}+a\right)+6 n\right] .
\end{gathered}
$$

Proof. Since $E_{\sigma_{1}}^{2}-E_{\sigma_{1}^{*}}^{2}=4 E_{2}\left(E_{3}+a\right)$ and $A_{3}+B_{3}+2 a=2\left(E_{2}+2 a\right)$, the first formula follows.

Similarly, since $A_{\sigma_{1}}^{3}-A_{\sigma_{1}^{*}}^{3}=8 E_{2}\left[E_{2}^{2}+3 \widetilde{A}_{2}\left(\widetilde{A}_{1}+a\right)\right]$ and $B_{\sigma_{1}}^{3}-B_{\sigma_{1}^{*}}^{3}=$ $8 E_{2}\left[E_{2}^{2}-3 \widetilde{B}_{2}\left(\widetilde{B}_{1}+a\right)\right]$, their sum is $8 E_{2}$ multiplied by

$$
2 E_{2}^{2}+3\left(\widetilde{A}_{2} \widetilde{A}_{1}-\widetilde{B}_{2} \widetilde{B}_{1}\right)+3 a\left(\widetilde{A}_{2}-\widetilde{B}_{2}\right) .
$$

But, $\widetilde{A}_{2} \widetilde{A}_{1}-\widetilde{B}_{2} \widetilde{B}_{1}=2\left(2 a E_{2}+n\right)$ and $\widetilde{A}_{2}-\widetilde{B}_{2}=2 a$. From this the second formula follows immediately. 
For the triple $\mathfrak{r}$, the first identity in the next property is

$$
\mathfrak{r}_{\sigma_{1}^{*}}^{4}+4=6^{4}+4=4 \cdot 13 \cdot 25=4\left(3^{2}+2^{2}\right)\left(3^{2}+4^{2}\right) .
$$

Property 43. For $a, b, t, m \in R$,

$$
\begin{gathered}
A_{\sigma_{1}^{*}}^{4}+4 a^{4}=4\left[\widetilde{A}_{2}^{2}+\widetilde{E}_{3}^{2}\right]\left[\widetilde{A}_{2}^{2}+\left(\widetilde{A}_{2}+a\right)^{2}\right], \\
B_{\sigma_{1}^{*}}^{4}+4 a^{4}=4\left[\widetilde{B}_{2}^{2}+\widetilde{E}_{3}^{2}\right]\left[\widetilde{B}_{2}^{2}+\left(\widetilde{B}_{2}-a\right)^{2}\right], \\
E_{\sigma_{1}}^{4}+4 a^{4}=4\left[\widetilde{E}_{1}^{2}+\left(\widetilde{E}_{1}+a\right)^{2}\right]\left[\widetilde{E}_{1}^{2}+\left(\widetilde{E}_{1}+3 a\right)^{2}-4 a^{2}\right] .
\end{gathered}
$$

Proof. Since $A_{\sigma_{1}^{*}}^{4}+4 a^{4}$ is $4 f g$, where $f=2 a m\left(\widetilde{A}_{2}+t\right)+(a+t)^{2}+t^{2}$ and $g=f+4 a \widetilde{A}_{2}$, the first formula follows because $f=\widetilde{A}_{2}^{2}+\widetilde{E}_{3}^{2}$ and $g=\widetilde{A}_{2}^{2}+$ $\left(\widetilde{A}_{2}+a\right)^{2}$.

\section{The GENERALIZED DETERMINANTS FROM COMPONENTS}

For $a=\left(a_{1}, a_{2}, a_{3}\right)$ and $b=\left(b_{1}, b_{2}, b_{3}\right)$ in $R^{3}$, let

$$
\begin{aligned}
& a \cdot b=\left|\begin{array}{ll}
a_{2} & a_{3} \\
b_{2} & b_{3}
\end{array}\right|+\left|\begin{array}{ll}
a_{3} & a_{1} \\
b_{3} & b_{1}
\end{array}\right|+\left|\begin{array}{ll}
a_{1} & a_{2} \\
b_{1} & b_{2}
\end{array}\right|, \\
& a: b=\left|\begin{array}{ll}
a_{2} & a_{3} \\
b_{2} & b_{3}
\end{array}\right|-\left|\begin{array}{ll}
a_{3} & a_{1} \\
b_{3} & b_{1}
\end{array}\right|+\left|\begin{array}{ll}
a_{1} & a_{2} \\
b_{1} & b_{2}
\end{array}\right| .
\end{aligned}
$$

Note that $a \cdot b$ is the determinant of the rectangular $2 \times 3$ matrix with rows $a$ and $b$ (see [14]). These generalized determinants are now tied with our triples $E$ and $\widetilde{E}$ using the following short notation.

Let $U=A \cdot \widetilde{A}, V=B \cdot \widetilde{B}, u=A \cdot \widetilde{B}, v=B \cdot \widetilde{A}, \ddot{U}=\widetilde{A}: A$, etc.

Property 44. For $a, b, t, m \in R$,

$$
\begin{aligned}
U=2 \widetilde{E}_{3}\left(E_{2}-a\right), & U+V=2\left(3 a \widetilde{B}_{1}+2 n\right), \\
V=2 \widetilde{B}_{2}\left(a-B_{3}\right), & U-V=2 \widetilde{B}_{1}\left(2 \widetilde{E}_{3}-a\right), \\
u-v=2 \widetilde{B}_{1}\left(2 \widetilde{E}_{3}+a\right), & u+v=\ddot{v}-\ddot{u}=-2\left(a \widetilde{B}_{1}+2 n\right) .
\end{aligned}
$$

Proof. Since $A \cdot \widetilde{A}$ is $2(a m+t)\left(a m^{2}+2 t m+b-a\right)$, we see that the first formula holds because $\widetilde{E}_{3}=a m+t$ and $E_{2}=a m^{2}+2 t m+b$.

Also, since $A \cdot \widetilde{B}-B \cdot \widetilde{A}$ is $2 f g$, where $f=a m^{2}-(a-2 t) m+b-t=\widetilde{B}_{1}$ and $g=2(a m+t)+a=\widetilde{E}_{3}+a$, it follows that the fifth formula also holds. 
Property 45. For $a, b, t, m \in R$,

$$
\begin{gathered}
\ddot{U}=2\left(\widetilde{A}_{1}^{2}+a \widetilde{A}_{1}+\widetilde{E}_{3}^{2}\right), \quad \ddot{V}=2 E_{2}\left(B_{3}-a\right), \\
\ddot{U}+\ddot{V}=2\left[2 E_{2}^{2}+a\left(3 \widetilde{A}_{1}-2 \widetilde{E}_{3}\right)+2 n\right], \\
\ddot{U}-\ddot{V}=2\left(4 \widetilde{A}_{1} \widetilde{E}_{3}-a \widetilde{B}_{1}-2 n\right), \\
\ddot{u}+\ddot{v}=2\left[2 E_{2}^{2}-a\left(A_{3}+\widetilde{B}_{2}\right)-2 n\right] .
\end{gathered}
$$

Proof. Since $\widetilde{B}: B$ is the product $2 f g$, where $f=a m^{2}+2 t m+b=E_{2}$ and $g=a m^{2}+2(t-a) m+b-2 t=B_{3}-a$, the second formula holds.

Similarly, the expression $\widetilde{A}: A-\widetilde{B}: B+2 a \widetilde{B}_{1}+4 n$ is the product $8(a m+$ $t)\left[a m^{2}+(a+2 t) m+b+t\right]$ of $8, \widetilde{E}_{3}$ and $\widetilde{A}_{1}$. This shows that the fourth formula holds.

\section{The sums of The PROduCts}

This section describes the three sets of identities on the factorizations for the sums of the products $\odot, \triangleright$ and $\triangleleft$ (from Section 9 ) of certain pairs from the triples $A, B, \widetilde{A}$ and $\widetilde{B}$. Let $P, Q, T$ and $\widetilde{T}$ be $A \odot \widetilde{A}, B \odot \widetilde{B}, A \odot B$ and $\widetilde{A} \odot \widetilde{B}$.

Property 46. For $a, b, t, m \in R$,

$$
\begin{gathered}
T_{\sigma_{1}}+2 n=2\left(\widetilde{A}_{1} \widetilde{B}_{1}+a^{2}\right), \quad T_{\sigma_{1}^{*}}+2 n=-2 \widetilde{A}_{2} \widetilde{B}_{2}, \\
\widetilde{T}_{\sigma_{1}}=E_{2}^{2}+\widetilde{A}_{2} \widetilde{B}_{2}, \quad \widetilde{T}_{\sigma_{1}^{*}}=E_{2}^{2}-\widetilde{A}_{2} \widetilde{B}_{2}, \\
P_{\sigma_{1}}=2 \widetilde{A}_{1} \widetilde{A}_{2}=2\left(A_{3} \widetilde{E}_{3}-n\right), \quad Q_{\sigma_{1}}=2 \widetilde{B}_{1} \widetilde{E}_{3}=2\left(E_{2} \widetilde{B}_{2}-n\right), \\
P_{\sigma_{1}^{*}}=2 \widetilde{A}_{2} \widetilde{E}_{3}=2\left(a \widetilde{A}_{1}+n\right), \quad Q_{\sigma_{1}^{*}}=-2 n .
\end{gathered}
$$

Proof. Since $T_{\sigma_{1}^{*}}+4 n$ is $2 a\left(a-a m^{2}-2 t m-b\right)=2 a\left(a-E_{2}\right)$, the second formula holds.

The product $Q$ has as the components the products $a\left[a m^{2}-(a-2 t) m+\right.$ $b-t],\left(a m^{2}+2 t m+b\right)(a m-a+t)$ and $\left[a m^{2}-2(a-t) m+a+b-2 t\right](a m+$ $t)$. Hence, $Q_{\sigma_{1}^{*}}$ is $2\left(a b-t^{2}\right)=-2 n$.

Property 47. For $a, b, t, m \in R$, if $M=A_{3} B_{3}+a^{2}$, then

$$
\begin{aligned}
& T_{\sigma_{2}}+a^{4}=M\left(E_{2}^{2}+a^{2}\right), \quad T_{\sigma_{2}^{*}}-a^{4}=M\left(E_{2}^{2}-a^{2}\right), \\
& \widetilde{T}_{\sigma_{2}}+n^{2}=2 E_{2}\left(E_{2}-a\right) \widetilde{E}_{3}^{2}, \quad \widetilde{T}_{\sigma_{2}^{*}}-n^{2}=2 a n E_{2} .
\end{aligned}
$$


Proof. The sum $T_{\sigma_{2}}+a^{4}$ factors as the product of $f$ and $g$, where $f$ is $a^{2} m^{4}$ $+4 a t m^{3}+2\left(2 t^{2}+a b\right) m^{2}+4 t b m+b^{2}+a^{2}=E_{2}+a^{2}$ and $g$ is $a^{2} m^{4}+$ $4 m t\left(a m^{2}-a+b\right)+2\left[a(b-a)+2 t^{2}\right] m^{2}+(a+b)^{2}+a^{2}-4 t^{2}=A_{3} B_{3}+a^{2}=M$. This proves the first formula.

The product $\widetilde{T}$ has as the components $\widetilde{A}_{1} \widetilde{B}_{1}, \widetilde{A}_{2} \widetilde{B}_{2}$ and $\widetilde{E}_{3}^{2}$. Hence, $\widetilde{T}_{\sigma_{2}^{*}}-n^{2}$ is the product 2 a $n E_{2}$, proving the last formula.

In the rest of this section, we consider the products $\triangleright$ and $\triangleleft$. Let $F=A \triangleright \widetilde{A}$, $G=A \triangleleft \widetilde{A}, H=B \triangleright \widetilde{B}$ and $K=B \triangleleft \widetilde{B}$.

Property 48. For $a, b, t, m \in R$, the following identities are true.

$$
\begin{gathered}
F_{\sigma_{1}}-G_{\sigma_{1}}=2 \widetilde{E}_{3}\left(E_{2}-a\right), \quad K_{\sigma_{1}}-H_{\sigma_{1}}=2 \widetilde{B}_{2}\left(B_{3}-a\right), \\
G_{\sigma_{2}}-F_{\sigma_{2}}=a E_{2}\left(E_{2}-a\right)\left(A_{3}+\widetilde{E}_{3}\right), \\
H_{\sigma_{2}}-K_{\sigma_{2}}=a B_{3}\left(B_{3}-a\right)\left(E_{2}+\widetilde{B}_{2}\right), \\
F_{\sigma_{1}^{*}}+G_{\sigma_{1}^{*}}=2 A_{3} \widetilde{A}_{2}, \quad H_{\sigma_{1}^{*}}-K_{\sigma_{1}^{*}}=2\left(B_{3}+\widetilde{E}_{3}\right)\left(B_{3}-a\right), \\
G_{\sigma_{2}^{*}}-F_{\sigma_{2}^{*}}=a E_{2}\left[A_{3}^{2}+\widetilde{E}_{3}\left(E_{2}-a\right)\right], \\
H_{\sigma_{2}^{*}}-K_{\sigma_{2}^{*}}=a B_{3}\left(B_{3}-a\right)\left(\widetilde{B}_{1}+a\right) .
\end{gathered}
$$

Proof. Since $F$ and $G$ are $\left(a \widetilde{A}_{2}, A_{2} \widetilde{A}_{3}, A_{3} \widetilde{A}_{1}\right)$ and $\left(a \widetilde{A}_{3}, A_{2} \widetilde{A}_{1}, A_{3} \widetilde{A}_{2}\right)$, we infer that the sums $F_{\sigma_{1}}$ and $G_{\sigma_{1}}$ are $A_{3}^{2}-a\left(3 \widetilde{A}_{1}-\widetilde{A}_{3}\right)-2 n$ and $\widetilde{A}_{1}^{2}+a\left(2 A_{3}\right.$ $-a)+n$. Hence, their difference is $2 \widetilde{E}_{3}\left(E_{2}-a\right)$. This proves the first identity. The proofs of the other seven identities are similar.

\section{THE PRODUCTS $\ltimes$ AND $\rtimes$}

Let $\ltimes$ and $\rtimes$ denote binary operations on $R^{3}$ defined by

$$
\begin{aligned}
& (a, b, c) \ltimes(d, e, f)=(b f-c e, c d-a f, a e-b d), \\
& (a, b, c) \rtimes(d, e, f)=(b f+c e, c d+a f, a e+b d) .
\end{aligned}
$$

Note that restricted on the standard Euclidean 3 -space $\mathbb{R}^{3}$ the product $\ltimes$ is the familiar vector cross-product.

In the next three properties we consider various symmetric sums of the products $\ltimes$ and $\rtimes$ when the fourth variable $m$ is either the sum $u+v$ or the difference $u-v$ of the elements $u$ and $v$ from the ring $R$.

Let $d=u-v, p=u v, p_{u}=p-u, p_{v}=p-v$ and $s=u+v$. Let $w=$ $a^{2} v^{2}-2 n$. We shall use $p, p_{u}$ and $p_{v}$ only in the last section.

Property 49. For $a, b, t, u, v \in R$,

$$
\begin{gathered}
\left(s^{A} \ltimes d^{A}\right)_{\sigma_{1}}=4 v\left(a^{2}-w+a u_{1}^{\widetilde{A}}\right), \quad\left(s^{E} \ltimes d^{E}\right)_{\sigma_{2}}=-(4 a v)^{2}(w+n), \\
\left(s^{B} \ltimes d^{B}\right)_{\sigma_{1}}=-\left(s^{\widetilde{B}} \ltimes d^{\widetilde{B}}\right)_{\sigma_{1}^{*}}=4 v\left(w-a^{2}-a u_{1}^{\widetilde{B}}\right) .
\end{gathered}
$$


Proof. Let $E=A$. We first notice that $s^{A} \ltimes d^{A}$ is $4 v(f, g,-h)$, where $f=$ $a u_{1}^{\widetilde{A}}-w, g=a u_{2}^{\widetilde{A}}$ and $h=a u_{3}^{\widetilde{A}}$. Hence, $\left(s^{A} \ltimes d^{A}\right)_{\sigma_{2}}$ is the product $-16 a^{2} v^{2}$ $\left(a^{2} v^{2}+a b-t^{2}\right)$. From this the second formula for $E=A$ follows easily.

Property 50. For $a, b, t, u, v \in R,\left(s^{\widetilde{A}} \ltimes d^{\widetilde{A}}\right)_{\sigma_{1}^{*}}=4 v\left(a u_{1}^{\widetilde{A}}-w\right)$, and

$$
\left(s^{\widetilde{A}} \ltimes d^{\widetilde{A}}\right)_{\sigma_{1}}=4 a v u_{3}^{\widetilde{E}}, \quad\left(s^{\widetilde{B}} \ltimes d^{\widetilde{B}}\right)_{\sigma_{1}}=-4 a v u_{2}^{\widetilde{B}} .
$$

Proof. Observe that $s^{\widetilde{A}} \ltimes d^{\widetilde{A}}$ is $-2 v(f, g, h)$, with $f=a^{2}, g=a u_{2}^{\widetilde{A}}-w$ and $h=w-a\left(u_{1}^{\widetilde{A}}+u_{2}^{\widetilde{A}}\right)$. Hence, $\left(s^{\widetilde{A}} \ltimes d^{\widetilde{A}}\right)_{\sigma_{1}}$ is $4 a v(a u+t)$. From this the second formula follows easily.

For the operation $\rtimes$ we have the following similar result. The right hand side of the first formula does not depend on $v$ and the right hand side of the second formula does not depend on $u$.

Property 51. For $a, b, t, u, v \in R$,

$$
\left(s^{\widetilde{A}} \rtimes d^{\widetilde{A}}\right)_{\sigma_{1}^{*}}+2 n=4 u_{2}^{\widetilde{A}} u_{3}^{\widetilde{A}}, \quad 2 n-\left(s^{\widetilde{B}} \rtimes d^{\widetilde{B}}\right)_{\sigma_{1}^{*}}=4 a^{2} v^{2} .
$$

Proof. We first notice that $s^{\widetilde{A}} \rtimes d^{\widetilde{A}}$ is a triple $(2 f, 2 g, 2 h)$, where $f=a u_{1}^{\widetilde{A}}-$ $n-w, g=u_{1}^{\widetilde{A}}\left(u_{2}^{\widetilde{A}}-a\right)-a v^{2} u_{2}^{\widetilde{A}}$ and $h=u_{1}^{\widetilde{A}} u_{2}^{\widetilde{A}}-a v^{2} u_{3}^{\widetilde{A}}$. From this we get $\left(s^{\widetilde{A}} \rtimes d^{\widetilde{A}}\right)_{\sigma_{1}^{*}}+2 n=4(a u+a+t)(a u+t)$ which is another way to write the first formula.

In the next property we collect formulas for the various symmetric sums of the products $F=A \ltimes B$ and $\widetilde{F}=\widetilde{A} \ltimes \widetilde{B}$.

Property 52. For $a, b, t, m \in R$ and $M=a \widetilde{E}_{3}+n, N=a E_{2} M$,

$$
\begin{gathered}
F_{\sigma_{1}}=4 \widetilde{E}_{3}\left(a-E_{2}\right), \quad \widetilde{F}_{\sigma_{1}}=-2 a \widetilde{B}_{1}, \quad F_{\sigma_{1}^{*}}=-4 \widetilde{E}_{3}\left(E_{2}+a\right), \\
\widetilde{F}_{\sigma_{1}^{*}}=2\left(a \widetilde{A}_{1}+2 n\right), \quad F_{\sigma_{2}}=F_{\sigma_{2}^{*}}=-16 a E_{2} \widetilde{E}_{3}^{2}, \\
\widetilde{F}_{\sigma_{2}}=-4\left(N+n^{2}\right), \quad \widetilde{F}_{\sigma_{2}^{*}}=-4\left(N-n^{2}+2 n M\right) .
\end{gathered}
$$

Proof. Since $F$ is $\left(-4 A_{2} \widetilde{A}_{3}, 4 a \widetilde{A}_{3}, 0\right)$, the first, the third and the fifth formulae follow easily.

Similarly, $\widetilde{F}$ is $\left(-2 a \widetilde{A}_{3}, 2 \widetilde{A}_{3}^{2},-2 n\right)$. Hence, the sum $\widetilde{F}_{\sigma_{1}}$ is $2 \widetilde{A}_{3}\left(\widetilde{A}_{3}-\right.$ a) $-2 n=2 a \widetilde{B}_{1}$. On the other hand, the sum $\widetilde{F}_{\sigma_{1}^{*}}$ is $2 \widetilde{A}_{3}\left(\widetilde{A}_{3}+a\right)+2 n$ $=2 a \widetilde{A}_{1}+4 n$. 


\section{Determinants AND THE PRODUCTS $\odot, \triangleright$ AND $\triangleleft$}

The first result in this section is another method to get the complete squares using the determinants and the products $\triangleright$ and $\triangleleft$.

Property 53. For $a, b, t, m \in R$,

$$
|A \triangleright B, B \triangleright B, A \triangleright A|=|A \triangleleft B, A \triangleleft A, B \triangleleft B|=\left[\begin{array}{lll}
4 a E_{2} & \widetilde{E}_{3}
\end{array}\right]^{2} .
$$

Proof. Since the rows of the matrix $M=[A \triangleright B, B \triangleright B, A \triangleright A]$ are the triples $\left(a E_{2}, E_{2} B_{3}, a A_{3}\right),\left(a E_{2}, E_{2} B_{3}, a B_{3}\right)$ and $\left(a E_{2}, E_{2} A_{3}, a A_{3}\right)$, by subtracting the first row from the second and the third, we infer that the matrix $M$ is equivalent to the matrix

$$
\left[\begin{array}{ccc}
a E_{2} & E_{2} B_{3} & a A_{3} \\
0 & 0 & a\left(B_{3}-A_{3}\right) \\
0 & E_{2}\left(A_{3}-B_{3}\right) & 0
\end{array}\right]
$$

Its determinant is obviously the square of $4 a E_{2} \widetilde{E}_{3}$. The determinant $\mid A \triangleleft B$, $A \triangleleft A, B \triangleleft B \mid$ is computed similarly.

We continue with two interesting results on determinants of matrices whose rows are certain products of pairs from the four pencils.

Property 54. For $a, b, t, m \in R$, the sum of $|A \triangleright B, B \odot B, A \odot A|$ and $\mid A \triangleleft$ $B, B \odot B, A \odot A \mid$ is the product $16 a E_{2} \widetilde{E}_{3}\left(E_{2}^{2}-a^{2}\right)\left(a+E_{2}-\widetilde{E}_{3}\right)$.

Proof. We leave the standard and tedious evaluation to the reader as a nice challenge.

Property 55. For $a, b, t, m \in R$,

$$
\begin{aligned}
&|\widetilde{A} \triangleleft \widetilde{B}, \widetilde{A} \triangleleft \widetilde{A}, \widetilde{B} \triangleleft \widetilde{B}|-|\widetilde{A} \triangleright \widetilde{B}, \widetilde{B} \triangleright \widetilde{B}, \widetilde{A} \triangleright \widetilde{A}|= \\
&|\widetilde{A} \odot \widetilde{B}, \widetilde{B} \odot \widetilde{B}, \widetilde{A} \odot \widetilde{A}|=8 a n \widetilde{E}_{3}^{3} .
\end{aligned}
$$

Proof. Note that

$$
M=[\widetilde{A} \odot \widetilde{B}, \widetilde{B} \odot \widetilde{B}, \widetilde{A} \odot \widetilde{A}]=\left[\begin{array}{ccc}
\widetilde{A}_{1} \widetilde{B}_{1} & \widetilde{A}_{2} \widetilde{B}_{2} & \widetilde{E}_{3}^{2} \\
\widetilde{B}_{1}^{2} & \widetilde{B}_{2}^{2} & \widetilde{E}_{3}^{2} \\
\widetilde{A}_{1}^{2} & \widetilde{A}_{2}^{2} & \widetilde{E}_{3}^{2}
\end{array}\right] .
$$

Now we subtract the first row of $M$ from the second and from the third. It follows that the matrix $M$ is equivalent with the matrix

$$
\left[\begin{array}{ccc}
\widetilde{A}_{1} \widetilde{B}_{1} & \widetilde{A}_{2} \widetilde{B}_{2} & \widetilde{E}_{3}^{2} \\
\widetilde{B}_{1}\left(\widetilde{B}_{1}-\widetilde{A}_{1}\right) & \widetilde{B}_{2}\left(\widetilde{B}_{2}-\widetilde{A}_{2}\right) & 0 \\
\widetilde{A}_{1}\left(\widetilde{A}_{1}-\widetilde{B}_{1}\right) & \widetilde{A}_{2}\left(\widetilde{A}_{2}-\widetilde{B}_{2}\right) & 0
\end{array}\right] .
$$


Since $\widetilde{A}_{1}-\widetilde{B}_{1}=2 \widetilde{E}_{3}, \widetilde{A}_{2}-\widetilde{B}_{2}=2 a$ and $\widetilde{A}_{1} \widetilde{B}_{2}-\widetilde{A}_{2} \widetilde{B}_{1}=2 n$, we see that $|\widetilde{A} \odot \widetilde{B}, \widetilde{B} \odot \widetilde{B}, \widetilde{A} \odot \widetilde{A}|=8$ an $\widetilde{E}_{3}^{3} . \quad$ Next, $|\widetilde{A} \triangleleft \widetilde{B}, \widetilde{A} \triangleleft \widetilde{A}, \widetilde{B} \triangleleft \widetilde{B}|$ and $\mid \widetilde{A} \triangleright \widetilde{B}$, $\widetilde{B} \triangleright \widetilde{B}, \widetilde{A} \triangleright \widetilde{A} \mid$ are evaluated similarly and one can check that their difference is again the product 8 an $\widetilde{E}_{3}^{3}$.

\section{SQUARES FROM DETERMINANTS AND PRODUCTS $\ltimes$ AND $\rtimes$}

In this section we shall describe three methods to get the complete squares using the determinants and the products $\ltimes$ and $\rtimes$.

Property 56. For $a, b, t \in R$ and $n=t^{2}-a b$,

$$
\left|(10 n)^{\widetilde{A}} \ltimes(10 n)^{\widetilde{B}},(4 n)^{\widetilde{A}} \ltimes(4 n)^{\widetilde{B}}, n^{\widetilde{A}} \ltimes n^{\widetilde{B}}\right|=[6 a n]^{4} .
$$

Proof. The product $(10 n)^{\widetilde{A}} \ltimes(10 n)^{\widetilde{B}}$ is the triple

$$
\left(2 a(10 a n+t),-2(10 a n+t)^{2}, 2 n\right) .
$$

The other two products have the same form with 10 replaced by 4 and 1 , respectively. By an easy calculation of the determinant of the above $3 \times 3$ matrix one finds that its value is indeed $(6 a n)^{4}$.

Property 57. For $a, b, t \in R$ and $m=a^{2}+2 n=a^{2}+2\left(t^{2}-a b\right)$,

$$
\left|(10 m)^{\widetilde{A}} \rtimes(-10 m)^{\widetilde{B}},(4 m)^{\widetilde{A}_{\rtimes}}(-4 m)^{\widetilde{B}}, m^{\widetilde{A}} \rtimes(-m)^{\widetilde{B}}\right|=\left[36 a t m^{2}\right]^{2} .
$$

Proof. The product $(10 m)^{\widetilde{A}} \rtimes(-10 m)^{\widetilde{B}}$ has as the components the products $2\left(t^{2}-10 a^{2} m-10^{2} a^{2} m^{2}\right), 2 t\left(b-10^{2} a m^{2}\right)$ and $2 t(b-a-2 \cdot 10 a m-$ $\left.10^{2} a \mathrm{~m}^{2}\right)$. The other two products have the same form with 10 replaced by 4 and 1 , respectively. Now it is easy to compute the determinant of the above $3 \times 3$ matrix and discover that its value is the square of $36 \mathrm{~atm}^{2}$.

Property 58. Let $R$ be a commutative ring with the multiplicative identity $e$. Let $a, b, t \in R$ and assume that $c$ is the inverse of $a$. If $m=2 c t$, then

$$
\begin{aligned}
& \left|(-m)^{A} \ltimes m^{A},(20 m)^{A} \ltimes(-20 m)^{B},(-2 m)^{A} \ltimes(2 m)^{B}\right|= \\
& \quad\left|(-m)^{B} \ltimes m^{B},(-20 m)^{A} \ltimes(20 m)^{B},(2 m)^{A} \ltimes(-2 m)^{B}\right|=\left(1056 t^{3}\right)^{2} .
\end{aligned}
$$

Proof. The products $(-m)^{A} \ltimes m^{A},(20 m)^{A} \ltimes(-20 m)^{B}$ and $(-2 m)^{A} \ltimes(2 m)^{B}$ are the triples $\left(8 t c\left(4 a^{2} c^{2} t^{2}-a t-2 t^{2}+a b\right),-8 a c t(a+t), 8 a c t^{2}\right),(4 t$ $\left.\left(1600 a c^{2} t^{2}+40 a c t-b\right), 4 a t(40 c t+1),-160 a c t^{2}\right)$ and $\left(4 t\left(16 a c^{2} t^{2}-\right.\right.$ $\left.4 a c t-b),-4 a t(4 c t-1), 16 a c t^{2}\right)$. The first above determinant has the value $11264 a^{2} c^{2} t^{6}\left(82 a^{2} c^{2}+18 a c-1\right)$. Since $a c=e$, it follows that this determinant is the square of $1056 t^{3}$. The proof for the second determinant is similar. 


\section{VALUES IN SUMS AND PRODUCTS}

In this section we shall show that the last variable $m$ has the property that the value of the sums $\sigma_{1}$ and $\sigma_{1}^{*}$ for the functions $\alpha, \beta, \widetilde{\alpha}$ and $\widetilde{\beta}$ in the quadruples $(a, b, t, u+v)$ and $(a, b, t, u v)$ can be recovered from their values at $(a, b, t, u)$ and $(a, b, t, v)$. The notation is from the Section 8 .

Property 59. For $a, b, t, u, v \in R$,

$$
\begin{gathered}
d s_{\sigma_{1}}^{E}-u u_{\sigma_{1}}^{E}+v v_{\sigma_{1}}^{E}=2 a d p, \quad d s_{\sigma_{1}^{*}}^{E}-u u_{\sigma_{1}^{*}}^{E}+v v_{\sigma_{1}^{*}}^{E}=0, \\
d s_{\sigma_{1}}^{\widetilde{E}}-u u_{\sigma_{1}}^{\widetilde{E}}+v v_{\sigma_{1}}^{\widetilde{E}}=d s_{\sigma_{1}^{*}}^{\widetilde{E}}-u u_{\sigma_{1}^{*}}^{\widetilde{E}}+v v_{\sigma_{1}^{*}}^{\widetilde{E}}=a d p .
\end{gathered}
$$

Proof. Let $E=A$. Since $d s_{\sigma_{1}}^{A}=2\left(u u_{1}^{\widetilde{A}}-v v_{1}^{\widetilde{A}}+a d p+a d\right), u u_{\sigma_{1}}^{A}=2 u\left(u_{1}^{\widetilde{A}}\right.$ $+a)$ and $v v_{\sigma_{1}}^{A}=2 v\left(v_{1}^{\widetilde{A}}+a\right)$, we obtain easily the first formula.

Similarly, since $d s_{\sigma_{1}^{*}}^{A}=2\left(u u_{2}^{\widetilde{A}}-v v_{2}^{\widetilde{A}}\right), u u_{\sigma_{1}^{*}}^{A}=2 u u_{1}^{\widetilde{A}}$ and $v v_{\sigma_{1}^{*}}^{A}=2 v v_{1}^{\widetilde{A}}$, we conclude that the second formula holds.

Property 60. For $a, b, t, u, v \in R$,

$$
\begin{gathered}
d p_{\sigma_{1}}^{E}-p_{v} u_{\sigma_{1}}^{E}+p_{u} v_{\sigma_{1}}^{E}=2 a d p_{u} p_{v}, \quad d p_{\sigma_{1}^{*}}^{E}-p_{v} u_{\sigma_{1}^{*}}^{E}+p_{u} v_{\sigma_{1}^{*}}^{E}=0, \\
d p_{\sigma_{1}}^{\widetilde{E}}-p_{v} u_{\sigma_{1}}^{\widetilde{E}}+p_{u} v_{\sigma_{1}}^{\widetilde{E}}=d p_{\sigma_{1}^{*}}^{\widetilde{E}}-p_{v} u_{\sigma_{1}^{*}}^{\widetilde{E}}+p_{u} v_{\sigma_{1}^{*}}^{\widetilde{E}}=a d p_{u} p_{v} .
\end{gathered}
$$

Proof. Let $E=A$. Define a function $f: R \rightarrow R$ by

$$
f(x)=a x^{2}+(a+2 t) x+a+b+t .
$$

Since $p_{\sigma_{1}}^{A}=2 f(p), u_{\sigma_{1}}^{A}=2 f(u)$ and $v_{\sigma_{1}}^{A}=2 f(v)$, we get

$$
d p_{\sigma_{1}}^{A}-p_{v} u_{\sigma_{1}}^{A}+p_{u} v_{\sigma_{1}}^{A}=2 a d p(u-1)(v-1)=2 a d p_{u} p_{v} .
$$

\section{REFERENCES}

[1] A. Baker and H. Davenport, The equations $3 x^{2}-2=y^{2}$ and $8 x^{2}-7=z^{2}$, Quart. J. Math. Oxford Ser. (2) 20 (1969), 129-137.

[2] G. E. Bergum and V. E. Hoggatt, A problem of Fermat and the Fibonacci sequence, Fibonacci Quart., 15 (1977), 323-330.

[3] E. Brown, Sets in which $x y+k$ is always a square, Math. Comput., 45 (1985), 613620.

[4] Z. Čerin and G. M. Gianella, On Diophantine triples from Pell and Pell-Lucas numbers, Acc. Sc. Torino - Atti Sci. Fis., 143 (2009), 83-94.

[5] Z. Cerin and G. M. Gianella, On D(-4) and D(8) triples from Pell and Pell-Lucas numbers, Rend. Circ. Mat. Palermo Suppl. N., 81 (2009), 73-83.

[6] Z. Čerin, On D(-1) and D(5) triples from Fibonacci and Lucas numbers, (preprint).

[7] Z. Čerin, Pencils of Euler triples, I, (preprint).

[8] Z. Čerin, On extended Euler quadruples, (preprint).

[9] http://www.math.hr/ duje/Diophantine m-tuples, References.html 
[10] A. Dujella, Generalized Fibonacci numbers and the problem of Diophantus, Fibonacci Quart., 34 (1996), 164-175.

[11] L. Euler, Commentationes Arithmeticae I, In Opera Omnia, Series I, volume II, B.G. Teubner, Basel, 1915.

[12] B. W. Jones, A variation on a problem of Davenport and Diophantus, Quart. J. Math. Oxford Ser. (2), 27 (1976), 349-353.

[13] L. Jones, A Polynomial Approach to a Diophantine Problem, Math. Mag., 72 (1999), $52-55$.

[14] M. Radić, A definition of determinant of rectangular matrix, Glasnik Mat., 1 (21) (1966), 17-22.

(Received: October 1, 2010)

(Revised: January 19, 2011)
Kopernikova 7

10010 Zagreb

Croatia

E-mail: cerin@math.hr 